\title{
Extinction of top-predator in a three-level food-chain model
}

\author{
Chuang-Hsiung Chiu ${ }^{1}$, Sze-Bi Hsu ${ }^{2}$
}

${ }^{1}$ General Education Center, Nantai Institute of Technology, 71008, Tainan, Taiwan

${ }^{2}$ Department of Mathematics, National Tsing Hua University, 30043, Hsinchu, Taiwan. e-mail: sbhsu@am.nthu.edu.tw

Received: 10 October 1997

Abstract. In this paper we extend the Lyapunov functions, constructed by A. Ardito and P. Ricciardi for predator-prey system [1], to the three level food chain models. We first consider a general three-level food-chain model. A criterion for the extinction of top predator will be given. Then we restrict our attentions to the case in which the prey is of logistic growth and predators have Holling's type II functional responses.

Key words: Food chain - Predator-prey model - Lyapunov function

\section{Introduction}

The main purpose of this paper is to establish the global stability for the case of extinction of top-predator of the following three-level food-chain model $[13,15,16]$ :

$$
\left\{\begin{array}{l}
\frac{d x}{d t}=\gamma x\left(1-\frac{x}{K}\right)-\frac{m_{1} x}{a_{1}+x} y, \\
\frac{d y}{d t}=\left(\frac{m_{1} x}{a_{1}+x}-d_{1}\right) y-\frac{m_{2} y}{a_{2}+y} z, \\
\frac{d z}{d t}=\left(\frac{m_{2} y}{a_{2}+y}-d_{2}\right) z, \\
x(0)=x_{0}>0, \quad y(0)=y_{0}>0, \quad z(0)=z_{0}>0,
\end{array}\right.
$$

where $\gamma, K>0, a_{i}, m_{i}, d_{i}>0, i=1$, 2. In (1.1) $x(t), y(t)$ and $z(t)$ represent the population density of prey, predator and top-predator at time $t$ respectively. The prey grows with intrinsic growth rate $\gamma$ and carrying capacity $K$ in the absence of predation. The predator (top-predator) consumes the prey 
(predator) with functional response of Michaelis-Menten type, $m_{1} x /$ $a_{1}+x\left(m_{2} y / a_{2}+y\right) . d_{1}, d_{2}$ are the death rates for predator, top-predator respectively.

The behavior of the solutions of three-level food-chain model (1.1) can be very complicated. System (1.1) has been studied by many authors recently [13, $15,16]$. They performed the normal form analysis for a degenerate equilibrium, planar Hopf and saddle node bifurcation analysis, to study the bifurcation phenomena in the absence of top-predator, and showed that the model (1.1) in some parameter range could have chaotic behavior by numerical simulation. Therefore it is not easy to complete the study for this three-level food-chain model. In this paper we shall restrict our attentions to the global stability of the equilibrium which represents the extinction of top-predator. We prove the main result by extending the Lyapunov functions introduced by A. Ardito and P. Ricciardi [1]. We discuss the problem for general three species food chain models, in particular, the case of the Holling's type III functional response.

The rest of this paper is organized as follows. In Sect. 2 we introduce a general three species food chain model. A criterion for the extinction of top-predator will be given. Examples with Holling's type III functional response for the top-predators are given to show the global stability. In Sect. 3 we prove our main result for system (1.1).

\section{General food-chain models}

Consider the following three species food chain model:

$$
\left\{\begin{array}{l}
\frac{d x}{d t}=x g(x)-p_{1}(x) y, \\
\frac{d y}{d t}=\left(p_{1}(x)-d_{1}\right) y-p_{2}(y) z, \\
\frac{d z}{d t}=\left(p_{2}(y)-d_{2}\right) z, \\
x(0)=x_{0}>0, \quad y(0)=y_{0}>0, \quad z(0)=z_{0}>0 .
\end{array}\right.
$$

The basic assumptions for (2.1) are:

(H1) $p_{i}(\cdot) \in C([0, \infty), \boldsymbol{R}), p_{i}(0)=0$ and $p_{i}^{\prime}(x)>0$ for all $x>0, i=1,2$.

(H2) There exists $x_{*}>0$ such that $p_{1}\left(x_{*}\right)=d_{1}$.

(H3) $g(\cdot) \in C([0, \infty), \boldsymbol{R})$ and there exits $K>x_{*}$ such that $g(K)=0$ and $g(x)(x-K)<0$ for all $x>0, x \neq K$.

Some typical forms of $p_{i}(x)$ and $g(x)$ could be found in $[4,8-10,12,14]$. For general three species food chain models, interested readers may consult $[5-7,11,17]$. It is easy to verify that the solutions $x(t), y(t), z(t)$ of model $(2.1)$ 
are bounded and $x(t) \leqq K$ for $t$ sufficiently large. When $z(t) \equiv 0$ in $(2.1)$, the prey isocline is

$$
y=h(x)=\frac{x g(x)}{p_{1}(x)}
$$

Under assumptions (H1) $-(\mathrm{H} 3)$, there exists an equilibrium $E_{*}=\left(x_{*}, y_{*}, 0\right)$ of (2.1) with $y_{*}=h\left(x_{*}\right)$. Define

$$
F(x)=\frac{y_{*}-h(x)}{\int_{x_{*}}^{x}\left[\left(p_{1}(\xi)-d_{1}\right) / p_{1}(\xi)\right] d \xi}:\left(0, x_{*}\right) \cup\left(x_{*}, K\right) \rightarrow \boldsymbol{R} .
$$

Theorem 2.1. Let $(H 1)-(H 3)$ hold and $(x(t), y(t), z(t))$ be the solution of system (2.1). If $p_{2}\left(y_{*}\right)-d_{2} \leqq 0$ and

(i) There exists $\theta>0$ such that

$$
\theta \geqq F(x) \text { for all } x \in\left(0, x_{*}\right) \text { and } \theta \leqq F(x) \text { for all } x \in\left(x_{*}, K\right) .
$$

(ii) There exists $c>0$ such that for all $y>0$ with $y \neq y_{*}$,

$$
\Delta(y)=c\left(p_{2}(y)-p_{2}\left(y_{*}\right)\right)-p_{2}(y) y^{\theta-1}\left(y-y_{*}\right)<0 .
$$

Then $(x(t), y(t), z(t)) \rightarrow\left(x_{*}, y_{*}, 0\right)$ as $t \rightarrow \infty$.

Proof. Let

$$
W(x, y, z)=\int_{y_{*}}^{y} s^{\theta-1}\left(s-y_{*}\right) d s+y^{\theta} \int_{x_{*}}^{x} \frac{p_{1}(\xi)-d_{1}}{p_{1}(\xi)} d \xi+c z .
$$

It is obvious that $W(x, y, z) \in C^{1}\left(\boldsymbol{R}_{+}^{3}, \boldsymbol{R}\right), W\left(x_{*}, y_{*}, 0\right)=0$ and $W(x, y, z)>0$ for $(x, y, z) \in \boldsymbol{R}_{+}^{3}-\left\{\left(x_{*}, y_{*}, 0\right)\right\}$. Then the derivative of $W$ along the trajectory of (2.1) is

Hence

$$
\begin{aligned}
\dot{W}(x, y, z)= & y^{\theta}\left(\frac{p_{1}(x)-d_{1}}{p_{1}(x)}\right)\left(x g(x)-p_{1}(x) y\right) \\
& +\left(y^{\theta}-y_{*} y^{\theta-1}+\theta y^{\theta-1} \int_{x_{*}}^{x} \frac{p_{1}(\xi)-d_{1}}{p_{1}(\xi)} d \xi\right) \\
& \times\left[\left(p_{1}(x)-d_{1}\right) y-p_{2}(y) z\right]+c\left(p_{2}(y)-d_{2}\right) z .
\end{aligned}
$$

$$
\begin{aligned}
\dot{W}(x, y, z)= & y^{\theta}\left(\frac{p_{1}(x)-d_{1}}{p_{1}(x)}\right)\left(x g(x)-p_{1}(x) y\right) \\
& +y^{\theta-1}\left(y-y_{*}+\theta \int_{x_{*}}^{x} \frac{p_{1}(\xi)-d_{1}}{p_{1}(\xi)} d \xi\right)\left[\left(p_{1}(x)-d_{1}\right) y-p_{2}(y) z\right] \\
& +c\left[\left(p_{2}(y)-p_{2}\left(y_{*}\right)\right)+\left(p_{2}\left(y_{*}\right)-d_{2}\right)\right] z
\end{aligned}
$$




$$
\begin{aligned}
& =y^{\theta}\left(p_{1}(x)-d_{1}\right)\left[h(x)-y_{*}+\theta \int_{x_{*}}^{x} \frac{p_{1}(\xi)-d_{1}}{p_{1}(\xi)} d \xi\right] \\
& \quad+c\left(p_{2}\left(y_{*}\right)-d_{2}\right) z-\theta y^{\theta-1} p_{2}(y) z \int_{x_{*}}^{x} \frac{p_{1}(\xi)-d_{1}}{p_{1}(\xi)} d \xi \\
& \quad+z\left[c\left(p_{2}(y)-p_{2}\left(y_{*}\right)\right)-y^{\theta-1} p_{2}(y)\left(y-y_{*}\right)\right] .
\end{aligned}
$$

From the assumption $p_{2}\left(y_{*}\right)-d_{2} \leqq 0$ and (2.2), (2.3), it follows that

$$
\dot{W}(x, y, z) \leqq 0 \quad \text { for } 0<x<K, y>0, z>0 .
$$

Hence we complete the proof of Theorem 2.1 by LaSalle's invariance principle $[17,18]$.

We observe that in order to satisfy (2.3), we must have

$$
c=\frac{p_{2}\left(y_{*}\right)}{p_{2}^{\prime}\left(y_{*}\right)} y_{*}^{\theta-1} .
$$

In the following we consider the three level food chain models similar to (1.1) except that the functional response for the top-predator is of Holling's type III.

Example 1. Let $p_{2}(y)=m_{2} y^{n} / a_{2}+y^{n}, n \in N, m_{2}>0, a_{2}>0$. From (2.4)

$$
c=\frac{a_{2}+y_{*}^{n}}{n a_{2}} y_{*}^{\theta} .
$$

Then from (2.3)

$$
\begin{aligned}
\Delta(y) & =\frac{a_{2}+y_{*}^{n}}{n a_{2}} y_{*}^{\theta}\left(\frac{m_{2} y^{n}}{a_{2}+y^{n}}-\frac{m_{2} y_{*}^{n}}{a_{2}+y_{*}^{n}}\right)-\frac{m_{2} y^{n}}{a_{2}+y^{n}} y^{\theta-1}\left(y-y_{*}\right) \\
& =\frac{m_{2} y_{*}^{\theta}}{n\left(a_{2}+y^{n}\right)}\left(y^{n}-y_{*}^{n}\right)-\frac{m_{2} y^{n}}{a_{2}+y^{n}} y^{\theta-1}\left(y-y_{*}\right) \\
& =\frac{m_{2}\left(y-y_{*}\right)}{a_{2}+y^{n}}\left[\frac{y_{*}^{\theta}}{n}\left(y^{n-1}+y^{n-2} y_{*}+\cdots+y y_{*}^{n-2}+y_{*}^{n-1}\right)-y^{\theta+n-1}\right] .
\end{aligned}
$$

If $0<y \leqq y_{*}$ then we have

$$
\begin{aligned}
& \frac{y_{*}^{\theta}}{n}\left(y^{n-1}+y^{n-2} y_{*}+\cdots+y y_{*}^{n-2}+y_{*}^{n-1}\right)-y^{\theta+n-1} \\
& \quad \geqq \frac{y^{\theta}}{n}\left(n y^{n-1}\right)-y^{\theta+n-1}=0 .
\end{aligned}
$$


If $y_{*}<y$ then we have

$$
\begin{aligned}
& \frac{y_{*}^{\theta}}{n}\left(y^{n-1}+y^{n-2} y_{*}+\cdots+y y_{*}^{n-2}+y_{*}^{n-1}\right)-y^{\theta+n-1} \\
& \quad \leqq \frac{y^{\theta}}{n}\left(n y^{n-1}\right)-y^{\theta+n-1}=0 .
\end{aligned}
$$

Hence $\Delta(y)<0$ for all $y>0$ with $y \neq y_{*}$. Then (2.3) in Theorem 2.1 are satisfied.

Example 2. Let $p_{2}(y)=m y^{2} /(a+y)(b+y), m>0, a>0$ and $b>0$. From (2.3), (2.4)

$$
c=\frac{\left(a+y_{*}\right)\left(b+y_{*}\right)}{(a+b) y_{*}+2 a b} y_{*}^{\theta} .
$$

and

$$
\begin{aligned}
\Delta(y)= & \frac{\left(a+y_{*}\right)\left(b+y_{*}\right)}{(a+b) y_{*}+2 a b} y_{*}^{\theta}\left(\frac{m y^{2}}{(a+y)(b+y)}-\frac{m y_{*}^{2}}{\left(a+y_{*}\right)\left(b+y_{*}\right)}\right) \\
& -\frac{m y^{2}}{(a+y)(b+y)} y^{\theta-1}\left(y-y_{*}\right) \\
= & \frac{m\left(y-y_{*}\right) y_{*}^{\theta}}{(a+y)(b+y)}\left(\frac{(a+b) y y_{*}+a b\left(y+y_{*}\right)}{(a+b) y_{*}+2 a b}\right) \\
& -\frac{m y^{2}}{(a+y)(b+y)} y^{\theta-1}\left(y-y_{*}\right) \\
= & \frac{m\left(y-y_{*}\right)}{(a+y)(b+y)}\left[\left(y+\frac{a b\left(y_{*}-y\right)}{(a+b) y_{*}+2 a b}\right) y_{*}^{\theta}-y^{\theta+1}\right] .
\end{aligned}
$$

If $0<y \leqq y_{*}$ then we have

$$
\left(y+\frac{a b\left(y_{*}-y\right)}{(a+b) y_{*}+2 a b}\right) y_{*}^{\theta}-y^{\theta+1} \geqq y y^{\theta}-y^{\theta+1}=0 .
$$

If $y_{*}<y$ then we have

$$
\left(y+\frac{a b\left(y_{*}-y\right)}{(a+b) y_{*}+2 a b}\right) y_{*}^{\theta}-y^{\theta+1} \leqq y y^{\theta}-y^{\theta+1}=0 .
$$

Hence $\Delta(y)<0$ for all $y>0$ with $y \neq y_{*}$. Then (2.3) in Theorem 2.1 are satisfied. 


\section{Main result}

If the top-predator is absent, i.e. $z(t) \equiv 0$, system (1.1) is reduced to

$$
\left\{\begin{array}{l}
\frac{d x}{d t}=\gamma x\left(1-\frac{x}{K}\right)-\frac{m_{1} x}{a_{1}+x} y, \\
\frac{d y}{d t}=\left(\frac{m_{1} x}{a_{1}+x}-d_{1}\right) y, \\
x(0)=x_{0}>0, \quad y(0)=y_{0}>0 .
\end{array}\right.
$$

System (3.1) has a unique equilibrium $E_{*}=\left(x_{*}, y_{*}\right)$ where $x_{*}=a_{1} d_{1} / m_{1}-d_{1}$, $y_{*}=\left(\gamma / d_{1}\right) x_{*}\left(1-\left(x_{*} / K\right)\right), m_{1}>d_{1}$. The behavior of the solutions of (3.1) is well known and is classified as followings [2-4]:

1. If $\left(K-a_{1}\right) / 2<x_{*}<K$, then $E_{*}$ is globally stable.

2. If $0<x_{*}<\left(K-a_{1}\right) / 2$, then $E_{*}$ is an unstable focus and it is surrounded by a unique stable limit cycle.

3. A supercritical Hopf bifurcation takes place at $x_{*}=\left(K-a_{1}\right) / 2$.

The prey isocline of system (3.1) is

$$
y=h(x) \frac{\gamma}{m_{1}}\left(1-\frac{x}{K}\right)\left(a_{1}+x\right) .
$$

For system (1.1), the function $F:\left(0, x_{*}\right) \cup\left(x_{*}, K\right) \rightarrow \boldsymbol{R}$ is given by

$$
\begin{aligned}
F(x) & =\frac{y_{*}-h(x)}{\int_{x_{*}}^{x}\left[\left(\frac{m_{1} \xi}{a_{1}+\xi}-d_{1}\right) /\left(\frac{m_{1} \xi}{a_{1}+\xi}\right)\right] d \xi} \\
& =\frac{y_{*}-h(x)}{\left(\frac{m_{1}-d_{1}}{m_{1}}\right)\left[x-x_{*}-x_{*} \ln \left(\frac{x}{x_{*}}\right)\right]} .
\end{aligned}
$$

Theorem 2.1 proposes a criterion, depending on a parameter $\theta$, for the extinction of top predator in three level food chain models, it is quite general and is applicable to most of food chains. To understand the meaning of $\theta$ geometrically, we observe that one branch of the graph of $y=F(x)$ lay above $y=\theta$, for $x \in\left(x_{*}, K\right)$ and the other lay below $y=\theta$, for $x \in\left(0, x_{*}\right)$. But it is not easy to verify the existence of $\theta$ satisfying the conditions in Theorem 2.1 for general three level food chain models. However, for system (1.1), we will show that the equilibrium $\left(x_{*}, y_{*}, 0\right)$ is globally stable, provided that $\left(x_{*}, y_{*}, 0\right)$ is locally stable (including neutrally stable case). The following is our main result for system (1.1).

Theorem 3.1. Let $(x(t), y(t), z(t))$ be the solution of system (1.1). If $\left(m_{2} y_{*} / a_{2}+y_{*}\right)-d_{2} \leqq 0$ and $\left(K-a_{1}\right) \leqq 2 x_{*}$ then $(x(t), y(t), z(t)) \rightarrow\left(x_{*}, y_{*}, 0\right)$ as $t \rightarrow \infty$. 
Proof. From (3.2), there exists $\bar{x}>\left(K-a_{1} / 2\right)$ such that $h(\bar{x})=h(0)=\gamma a_{1} / m_{1}$. Consider the following cases

Case (i): $\left.\left(\left(K-a_{1}\right) / 2\right)\right)<x_{*} \leqq \bar{x}$.

There exists $0 \leqq \hat{x}<\left(K-a_{1}\right) / 2$, such that $h(\hat{x})=y_{*}$, then from (3.3), $F(x)$ satisfies:

$$
\begin{aligned}
& \lim _{x \rightarrow 0^{+}} \quad F(x)=0, \quad F(\hat{x})=0, \lim _{x \rightarrow x_{*}^{-}} F(x)=-\infty, \quad \lim _{x \rightarrow x_{*}^{-}} F(x)=\infty \text { and } \\
& F(x)>0 \quad \text { for } 0<x<\hat{x}, \quad F(x)<0 \quad \text { for } \hat{x}<x<x_{*} \text { and } \\
& F(x)>0 \quad \text { for } x_{*}<x \leqq K .
\end{aligned}
$$

Claim:

$$
\max _{0 \leqq x \leqq \hat{x}} F(x) \leqq \min _{x_{*}<x \leqq K} F(x) .
$$

If (3.4) does not hold, then there exists $\beta>0$ such that the equation $F(x)=\beta$ has three distinct roots $\delta_{1}, \delta_{2}, \delta_{3}$ satisfying $0<\delta_{1}<\delta_{2}<\hat{x}<\delta_{3} \leqq K$. Consider the function

$$
H(x)=y_{*}-h(x)-\beta\left(\frac{m_{1}-d_{1}}{m_{1}}\right)\left(x-x_{*}-x_{*} \ln \left(\frac{x}{x_{*}}\right)\right) .
$$

Then $H(x)=0$ has four roots namely $x_{*}$ and $\delta_{1}, \delta_{2}, \delta_{3}$ in $[0, K]$. Since

$$
H^{\prime \prime \prime}(x)=2 \frac{\beta\left(m_{1}-d_{1}\right) x_{*}}{m_{1} x^{3}},
$$

from Rolle's Theorem, there exists $\eta \in(0, K)$ such that $H^{\prime \prime \prime}(\eta)=0$ which is obviously a contradiction to (3.5). Hence (3.4) holds. From (3.4) we choose $\theta>0$ s£Luch that

$$
\max _{0 \leqq x \leqq \hat{x}} F(x) \leqq \theta \leqq \min _{x_{*}<x \leqq K} F(x) .
$$

Thus $\theta$ satisfies the hypothesis in Theorem 2.1, and $(x(t), y(t), z(t)) \rightarrow\left(x_{*}, y_{*}, 0\right)$ as $t \rightarrow \infty$.

Case (ii): $\left(\left(K-a_{1}\right) / 2\right)<\bar{x} \leqq x_{*}$.

From (3.3), $F(x)$ satisfies:

$$
\begin{aligned}
& F(x)<0 \quad \text { for } 0<x<x_{*}, \quad \lim _{x \rightarrow 0^{+}} F(x)=0, \quad \lim _{x \rightarrow x_{*}^{-}} F(x)=-\infty \text { and } \\
& F(x)>0 \quad \text { for } x_{*}<x \leqq K, \quad \lim _{x \rightarrow x_{*}^{+}} F(x)=\infty .
\end{aligned}
$$

Obviously the hypothesis in Theorem 2.1 is satisfied with $\theta$ satisfying

$$
0<\theta<\min _{x_{*}<x \leqq K} F(x) .
$$

Case (iii): $x_{*}=\left(\left(K-a_{1}\right) / 2\right)$. 
From (3.3), $F(x)$ satisfies:

and

$$
\lim _{x \rightarrow 0^{+}} F(x)=0, \quad \lim _{x \rightarrow x^{+}} F(x)=\frac{\gamma\left(K-a_{1}\right)}{K\left(m_{1}-d_{1}\right)},
$$

$$
F(x)>0 \text { for } 0<x \leqq K .
$$

Therefore $F(x)$ is continuous in $(0, K]$. Let $x=\eta x_{*}, \eta \in(0,1) \cup\left(1, K / x_{*}\right)$, then from (3.2) we have

$$
\begin{gathered}
h\left(\eta x_{*}\right)=\frac{\gamma}{m_{1} K}\left[-(\eta-1)^{2} x_{*}^{2}+x_{*}^{2}+K a_{1}\right], \\
y_{*}=h\left(x_{*}\right)=\frac{\gamma}{m_{1} K}\left(x_{*}^{2}+K a_{1}\right) .
\end{gathered}
$$

From (3.3) we have

$$
\begin{aligned}
F(x) & =F\left(\eta x_{*}\right)=\frac{y_{*}-h\left(\eta x_{*}\right)}{\left(\frac{m_{1}-d_{1}}{m_{1}}\right)\left(\eta x_{*}-x_{*}-x_{*} \ln \eta\right)} \\
& =\frac{\gamma x_{*}}{K\left(m_{1}-d_{1}\right)}\left(\frac{(\eta-1)^{2}}{\eta-1-\ln \eta}\right), \quad \eta \in(0,1) \cup\left(1, \frac{K}{x_{*}}\right) .
\end{aligned}
$$

Consider the following function

$$
f(\eta)=\eta^{2}+2 \ln \eta-4 \eta+3, \quad \eta \in\left(0, \frac{K}{x_{*}}\right) .
$$

Obviously,

$$
\lim _{\eta \rightarrow 0^{+}} f(\eta)=-\infty, \quad f(1)=0 \quad \text { and } \quad f^{\prime}(\eta)=\frac{2(\eta-1)^{2}}{\eta}>0, \quad \forall \eta \in\left(0, \frac{K}{x_{*}}\right) .
$$

Hence $f(\eta)$ is an increasing function. If $\eta \in(0,1)$ then $x \in\left(0, x_{*}\right)$, and we have

This implies

$$
\eta^{2}+2 \ln \eta-4 \eta+3<0 \text {. }
$$

$$
\frac{(\eta-1)^{2}}{\eta-1-\ln \eta}<2
$$

hence from (3.6) we have

$$
F(x)<\frac{2 \gamma x_{*}}{K\left(m_{1}-d_{1}\right)}=\frac{\gamma\left(K-a_{1}\right)}{K\left(m_{1}-d_{1}\right)}, \quad \forall x \in\left(0, x_{*}\right) .
$$

If $\eta \in\left(1, K / x_{*}\right)$ then $x \in\left(x_{*}, K\right)$, and we have

This implies

$$
\eta^{2}+2 \ln \eta-4 \eta+3>0 \text {. }
$$

$$
\frac{(\eta-1)^{2}}{\eta-1-\ln \eta}>2
$$


hence from (3.6) we have

$$
F(x)>\frac{2 \gamma x_{*}}{K\left(m_{1}-d_{1}\right)}=\frac{\gamma\left(K-a_{1}\right)}{K\left(m_{1}-d_{1}\right)}, \quad \forall x \in\left(x_{*}, K\right) .
$$

From (3.7), (3.8) we choose $\theta=\gamma\left(K-a_{1}\right) / K\left(m_{1}-d_{1}\right)$. Thus $\theta$ satisfies the hypothesis in Theorem 2.1, and $(x(t), y(t), z(t)) \rightarrow\left(x_{*}, y_{*}, 0\right)$ as $t \rightarrow \infty$. Therefore we complete the proof of Theorem 3.1.

\section{References}

1. A. Ardito and P. Ricciardi, Lyapunov functions for a generalized Gause-type model, J. Math. Biol. 33, 816-828 (1995)

2. G. J. Butler, S. B. Hsu and P. Waltman, Coexistence of competing predators in a chemostat, J. Math. Biol. 17, 133-151 (1983)

3. K. S. Cheng, Uniqueness of a limit cycle of a predator-prey system, SIAM J. Math., Anal. 12, 541-548 (1981)

4. K. S. Cheng, S. B. Hsu and S. S. Lin, Some results on global stability of predator-prey system, J. Math. Biol. 12, 115-126 (1981)

5. H. I. Freedman and W. H. So, Global stability and persistence of simple food chains, Math. Biosci. 76, 69-86 (1985)

6. H. I. Freedman and P. Waltman, Mathematical analysis of some three species food chain models, Math. Biosci. 33, 257-276 (1977)

7. H. I. Freedman and P. Waltman, Persistence in models of three interacting predator-prey population, Math. Biosci. 68, 213-231 (1984)

8. B. S. Goh, Global stability in two species interaction, J. Math. Biol. 3, 313-318 (1976)

9. G. W. Harrion, Global stability of a predator-prey interaction, J. Math. Biol. 8, 159-171 (1979)

10. S. B. Hsu, On global stability of a predator-prey system, Math. Biosci. 39, 1-10 (1978)

11. S. B. Hsu, S. P. Hubbell and P. Waltman, Competing predators, SIAM J. Appl. Math., 35(4), 617-625 (1978).

12. S. B. Hsu and T. W. Huang, Global stability for a class of predator-prey systems, SIAM J. Appl. Math., 55(3), 763-783 (1955)

13 A. Klebanoff and A. Hastings, Chaos in three species food chains, J. Math. Biol. 32, 427-451 (1994)

14. Y. Kuang, Global stability of Gause-type predator-prey systems, J. Math. Biol. 28, 463-474 (1990)

15. Yu. A. Kuznetsov and S. Rinaldi, Remarks on food chain dynamics, Math. Biosic. 134, 1-33 (1996)

16. K. McCann and P. Yodzis, Bifurcation structure of a three-species food chain model, Theor. Pop. Biol. 48, 93-125 (1995)

17. Hal I. Smith and P. Waltman, The theory of the chemostat, Cambridge University Press (1995)

18. G. Wolkowicz and Z. Lu, Global dynamics of a mathematical model of competition in Zthe chemostat: general response functiion and different death rates, SIAM J. Appl. Math. 52(1), 222-233 (1992) 